Research Article

\title{
Discrete Element Simulation of Factors Affecting the Fluidity of Nylon Powder
}

\author{
Shengqiang Jiang $\mathbb{D}^{1,2}$ Chunyan Duan $\mathbb{D}^{1,2}$ Yixuan Ye ${ }^{10},,^{1,2}$ Chao Tang $\mathbb{D}^{1,2}$ \\ and Xiaodong Chen $\mathbb{1 D}^{1,2}$ \\ ${ }^{1}$ School of Mechanical Engineering, Xiangtan University, Xiangtan 411105, China \\ ${ }^{2}$ Engineering Research Center of Complex Tracks Processing Technology and Equipment of Ministry of Education, \\ Xiangtan 411105, China
}

Correspondence should be addressed to Shengqiang Jiang; jsqcx@xtu.edu.cn

Received 5 June 2019; Accepted 22 July 2019; Published 25 August 2019

Guest Editor: Qijun Zheng

Copyright (c) 2019 Shengqiang Jiang et al. This is an open access article distributed under the Creative Commons Attribution License, which permits unrestricted use, distribution, and reproduction in any medium, provided the original work is properly cited.

\begin{abstract}
Powder fluidity is one of the important factors affecting the smoothness of selective laser sintered parts and the mechanical properties of sintered parts. In this paper, the angle of repose (AOR) and angle of internal friction of nylon PA3200 powder were measured by a powder comprehensive tester and a direct shear tester to evaluate the flow properties of the powder particles. Based on the discrete element method (DEM), the rolling resistance contact model and the van der Waals force model were used to describe the interaction forces between the powder particles. The rolling resistance coefficient and friction coefficient within the contact model were calibrated by the results of the AOR experiments. Based on the orthogonal experimental method, the particle size and particle size distribution (PSD) (such as uniform distribution and Gaussian distribution) were selected as the influencing factors, and the effect of particle size and PSD on the fluidity of nylon PA3200 powder was studied by numerical simulation. The results show that the PSD has a stronger influence on the AOR than particle size, and the fluidity of uniform distribution is better than that of the Gaussian distribution.
\end{abstract}

\section{Introduction}

Selective laser sintering (SLS) is a rapid prototyping technology based on the principle of additive manufacturing, which makes parts by layering the powder and scanning sintering layer by layer. The study of powder fluidity is the most basic content in powder engineering and is also an important part of SLS forming research [1]. Before the process of laser scanning sintering, the powder paving process is very important to the manufacturing process of SLS, as shown in Figure 1. Bad powder fluidity will lead to problems of poor spreading performance of the powder during the laying process, uneven density distribution of the powder layer, and warping and holes of the sintering layer during sintering, and finally the size accuracy and mechanical properties of the sintered parts cannot meet the predetermined requirements. On the contrary, the better the fluidity of the powder is, the better its spreading performance and subsequent sintering quality are. Hence, it is important to study the flow abilities of the powder for the SLS process.

Powder fluidity has an obvious multiscale effect [2], which is closely related to powder physical property parameters (such as particle size, density, and particle shape). The friction of adhesion between particles, van der Waals force, electrostatic force, and other factors hinder the free flow of particles and affect the fluidity of powder. Yang and Evans [3] studied the effects of sphericity and particle size on the fluidity of the powder. The results show that the higher the sphericity in the powder, the better the fluidity, and the larger size particle has a better fluidity than the smaller one. Goh et al. [4] compared the powder flow parameters with the particle size and shape. They found that the smallest fraction of particle size has an important influence on most of the flow parameters because of its cohesiveness, while the particle shape was important for flow parameters which were more sensitive to interparticulate friction. Zhang et al. [5, 6] studied the correlation of supercritical water (SCW) from 


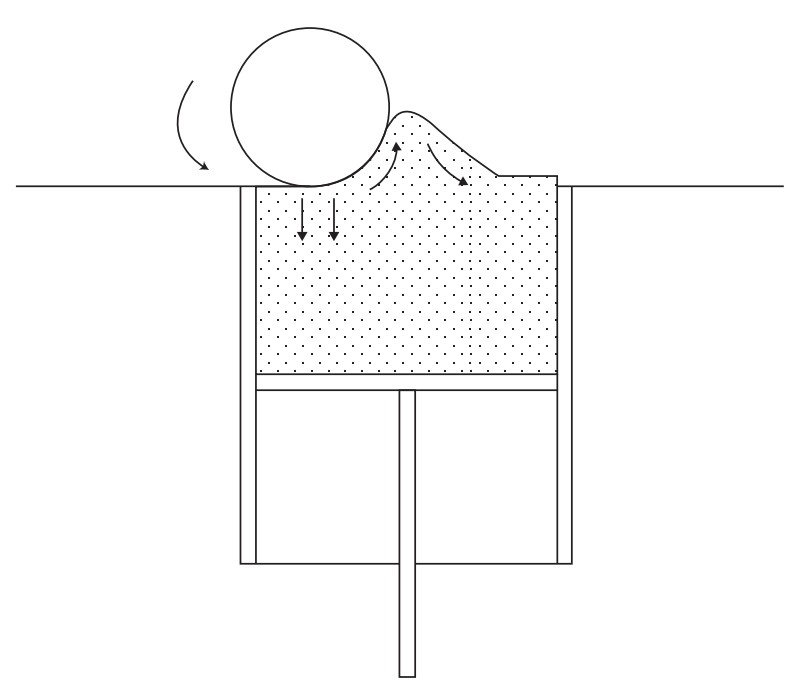

FIgURE 1: Schematic diagram of roller dusting.

the aspect ratio of the ellipsoid, showing that particle shape has a great influence on the research results. Wei et al. [7] demonstrated that shape has a certain influence on the stability of filling structure of particles and the uniformity of pore distribution. Dai et al. [8] found that the sliding friction, rolling friction, and PSD all have a strong influence on the structural characteristics of the repose angle of sand piles. Macías-García et al. [9] proposed a Rosin-Rammler distribution for describing the PSD based on probability and statistical theory and obtained the uniformity coefficient to describe the size distribution range of powder. Fitzpatrick et al. [10] have found that the smaller the powder particles, the worse the fluidity. They found the reason is that as the size of the particle becomes smaller and the specific surface area was increased, the viscous force between the particles becomes larger to prevent the flow of the powder. Yu et al. [11] found a certain relationship between the deposition state of the powder and the interaction between the particles and the particle size. Amorós et al. [12] studied the monodisperse quartz sand with different PSDs as materials to test the relationship between particles, particle size, and bed tightness, and the results showed that the cohesive force increases with the increase of the tightness and the decrease of the particle size. Wang et al. [13] measured the AOR of coal powder with different particle sizes by three methods, and the results showed that when the particle size was larger than a certain critical size, the fluidity was better with the increase of the particle size of pulverized coal; when it was smaller than the critical size, the situation would be opposite. Al-Hashemi and Al-Amoudi [14] showed that the AOR of particles is an important parameter to understand the microscopic behavior of granular materials and to relate them to macroscopic behavior.

It can be seen from the above studies that the physical parameters of powders have an important influence on the fluidity of powders, and the modification of powders can improve the fluidity of powders to a certain extent. However, there are few studies on the fluidity of nylon PA3200 powder used in SLS process, and most of the powder fluidity is in the observation stage of the test phenomenon. In this paper, nylon PA3200 powder was taken as the research object and a series of experiments and numerical simulations were used to study the influencing factors of nylon PA3200 powder flow performance. The effects of physical properties of nylon PA3200 powder on its fluidity were studied.

\section{Characterization and Experiment of Powders}

2.1. Powder Characterization. The particle size and surface morphology of the powder have a great influence on the microscopic force of the powder. Therefore, the particle size test and surface topography of the nylon PA3200 powder are required. The nylon powder materials used in the experimental study were produced by Hunan Farsoon HighTechnology Co., Ltd., China.

2.1.1. Particle Size Measurement. In this paper, the particle size of nylon PA3200 powder was tested by a laser particle size analyzer (Mastersizer 2000, Malvern, UK). The PSD results as shown in Figure 2 were obtained. It can be seen from the figure that the PSD of nylon PA3200 powder is in the range of $20-120 \mu \mathrm{m}$, and it is normally distributed. The measurement results show that D90 is $90 \mu \mathrm{m}, \mathrm{D} 50$ is $49.5 \mu \mathrm{m}$, and D10 is $25.6 \mu \mathrm{m}$ (D90, D50, and D10 represent a cumulative distribution curve; distribution of the powder was $90 \%, 50 \%$, and $10 \%$ of the equivalent diameter, respectively).

2.1.2. Observation of Surface Topography. The microscopic morphology of nylon PA3200 powder was observed by ultradepth of field microscope (VHX-2000c, Keyence, Japan). As shown in Figure 3, it can be seen that most powder morphologies are approximately spherical with a certain degree of agglomeration. Referring to this microscopic morphology, a spherical model was used to simulate nylon PA3200 powder.

2.2. Powder Fluidity Experiment. The powder is a collection of particles in a discrete state. However, there is no clear definition of the fluidity of the powder, and there is no uniform measurement method. Most of the existing research studies describe the flow properties of the powder through macroscopic accumulation and flow experiments, such as the AOR method [15], the Hausner index method [16], the Carr flow index method [17], and the uniaxial compression test [18]. The parameters characterized generally include the AOR, the internal friction of angle, and the cohesion. This paper mainly measures the AOR, angle of internal friction, and coefficient of internal friction.

2.2.1. Measurement of the AOR. The AOR refers to the angle between the powder stacking slope and the bottom horizontal plane in a static equilibrium state. It is formed by a specific way to naturally drop the powder onto a specific platform. The schematic diagram is shown in Figure 4. In general, the smaller the AOR is, the smaller the friction between the 


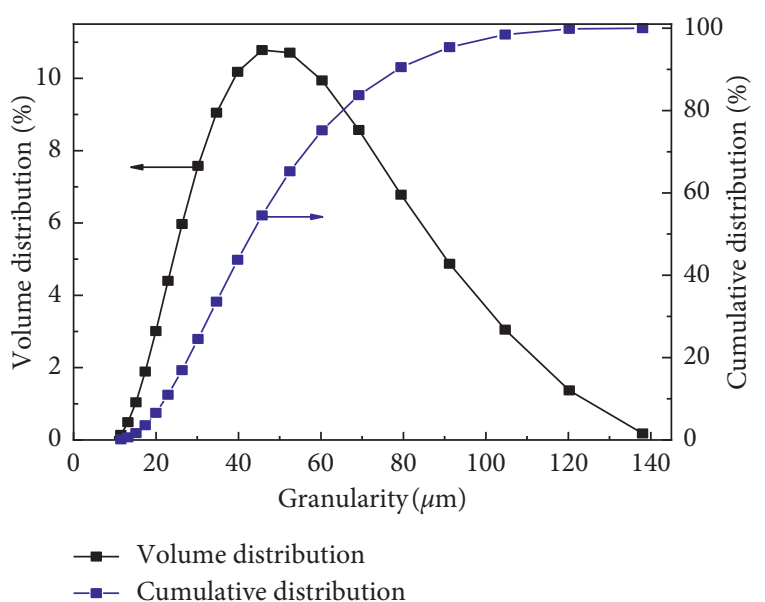

FIGURE 2: PSD measurement results. The black curve represents the percentage of particles of different particle sizes to the total volume, and the blue curve represents the cumulative volume percentage of particles.

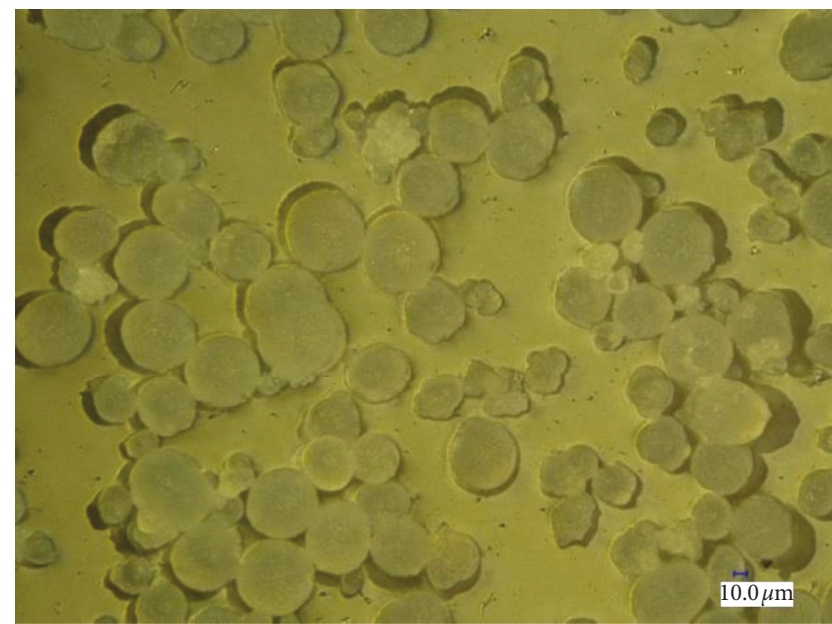

Figure 3: Microstructure of nylon PA3200 powder.

powders is, and the better the fluidity of the powder is. In this paper, the powder angle comprehensive tester (BT-1000, Dandong Baite Instrument Co., Ltd., China) was used to determine the AOR for nylon PA3200 powder. Since the AOR measured by different positions of the nylon PA3200 powder pile may be different, the angles of repose under three different positions of the pile (such as $0^{\circ}, 120^{\circ}$, and $240^{\circ}$ ) were measured in each set of experiments to reduce the experimental error. The average value of the AOR was computed, as shown in Table 1 , and the average AOR is $35.49^{\circ}$.

2.2.2. Measurement of the Friction Angle and Internal Friction Coefficient of the Powder. In this paper, the straincontrolled direct shear instrument (ZJ, Nanjing Soil Instrument Factory, China) was used to determine the internal friction angle and internal friction coefficient of nylon PA3200 powder. According to the test method of the shear instrument using the Jenike method [19], as shown in Figure 5, nylon PA3200 powders were shear tested under

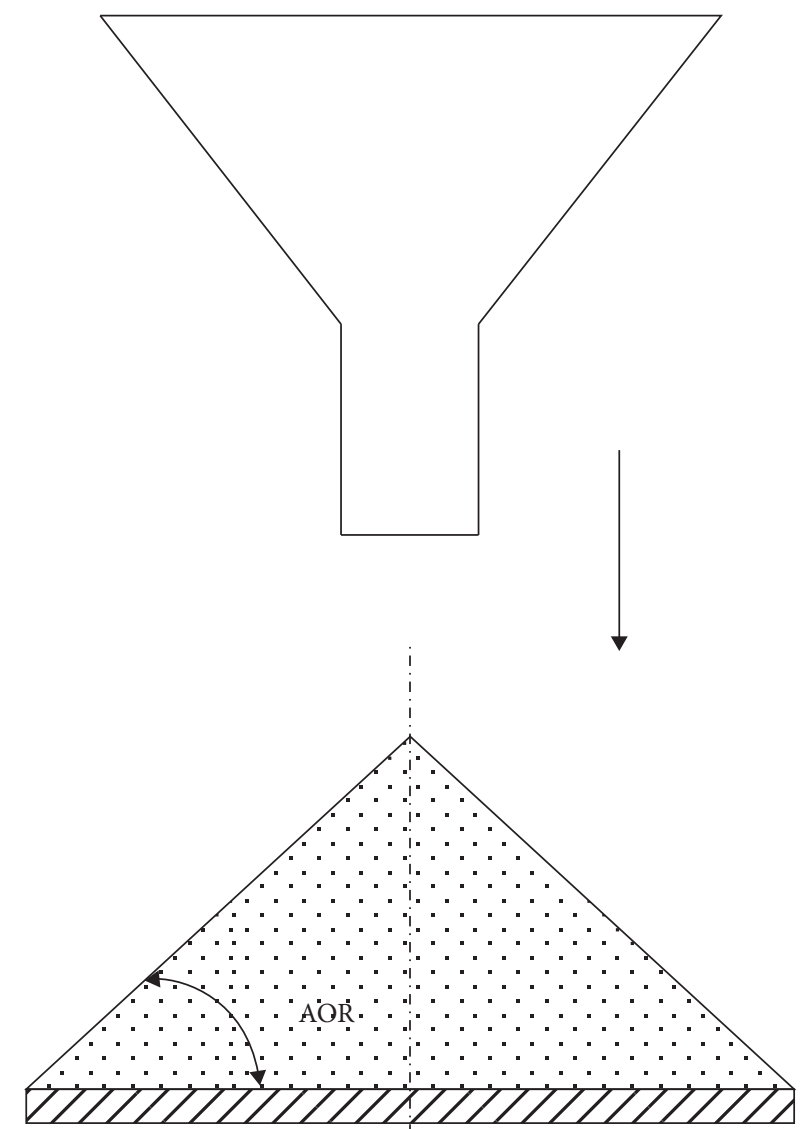

FIgURE 4: Schematic diagram of the AOR.

TABLE 1: Measured results of the AOR with nylon PA3200 powder.

\begin{tabular}{lccccc}
\hline $\begin{array}{l}\text { Number of } \\
\text { experiments }\end{array}$ & $0^{\circ}$ & $120^{\circ}$ & $240^{\circ}$ & $\begin{array}{r}\text { Average } \\
\text { value }\left(^{\circ}\right)\end{array}$ & $\begin{array}{c}\text { AOR } \\
\left({ }^{\circ}\right)\end{array}$ \\
\hline 1 & 36.1 & 35.3 & 35.3 & 35.57 & \\
2 & 36.5 & 35.1 & 34.8 & 35.47 & 35.49 \\
3 & 36.2 & 35.6 & 34.5 & 35.43 & \\
\hline
\end{tabular}

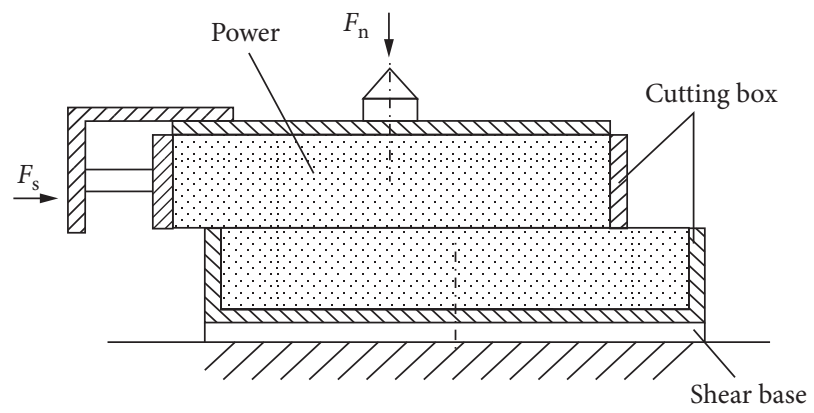

Figure 5: Schematic diagram of the shearing process $\left(F_{\mathrm{n}}\right.$ is the vertical pressure and $F_{\mathrm{s}}$ is the shear force).

four different vertical pressures, which were $100 \mathrm{kPa}$, $200 \mathrm{kPa}, 300 \mathrm{kPa}$, and $400 \mathrm{kPa}$, respectively. The working principle is that the powder was placed in the shear box, and then a certain vertical pressure was applied to the powder, and then a horizontal thrust was applied to the shear box 
below, making the powder shear displacement along the horizontal contact surface of the upper and lower boxes. In the process of shearing, the shear stress on the cross section of the powder can be calculated by measuring the horizontal thrust applied at every fixed time, that is, the increment of shear deformation at every fixed value. Determine the corresponding internal friction angle $\varphi$ to characterize the powder flow properties. The shear force $\tau$ of the specimen when the shear failure occurs can be calculated as follows:

$$
\tau=C R,
$$

where $C$ is the force ring coefficient $(\mathrm{kPa} / 0.01 \mathrm{~mm})$ and $R$ is the dynamometer reading $(0.01 \mathrm{~mm})$.

Figure 6(a) shows the relationship between shear stress and shear displacement of nylon PA3200 powder. As the shear displacement increases, the shear force tends to be stable, indicating that the powder has undergone shear failure. Extracting the maximum shear forces $S_{1}, S_{2}, S_{3}$, and $S_{4}$ at the time of shear failure, and the shear strength and vertical pressure relationship curves are shown in Figure 6(b). The equation can be obtained by fitting the curve as follows:

$$
\begin{aligned}
\tau & =\mu \sigma-\alpha, \\
\mathrm{AOR} & =\arctan \mu,
\end{aligned}
$$

where $\mu$ is the internal friction coefficient and $\sigma$ and $\alpha$ are the constant parameters obtained by fitting the curve.

The internal friction coefficient and the friction angle were calculated to be 0.50 and $26.57^{\circ}$, respectively. Through the above experiments, the macroscopic characteristic parameters of nylon PA3200 powder were determined, as shown in Table 2.

\section{DEM Contact Model}

The discrete element method (DEM) was first proposed by Cundall and Strack [20] in the later 1970s. Based on Newton's second law of motion, this method predicts particle swarm behavior by calculating the interaction and relative motion between particles at each moment [21] and was first used in the analysis of rock mechanics problems. The basic idea of the DEM is to separate the discontinuous body into a set of rigid elements so that each rigid element satisfies the equation of motion and solve the equation of motion of each rigid element by a time-step iterative method to obtain the overall motion form of the discontinuous body. The macroscopic mechanical behavior of the material is achieved by the constitutive model of each contact. Therefore, the selection of the contact model is crucial for the establishment of the DEM model. This section introduces the contact force model of nylon PA3200 powder.

In DEM, each particle is tracked and its motion is governed by Newton's second law [22]:

$$
\begin{aligned}
& \text { translational motion: } m \frac{d \mathbf{V}_{i}}{d t}=\mathbf{F}_{i}-\beta_{\mathrm{g}} \mathbf{V}_{i}, \\
& \text { rotational motion: } I \frac{d \boldsymbol{\omega}_{i}}{d t}=\mathbf{M}_{i}-\beta_{\mathrm{g}} \boldsymbol{\omega}_{i},
\end{aligned}
$$

where $i(=1,2,3)$ denotes the $x, y$, and $z$ coordinate directions, respectively; $\mathbf{F}_{i}$ is the out-of-balance force

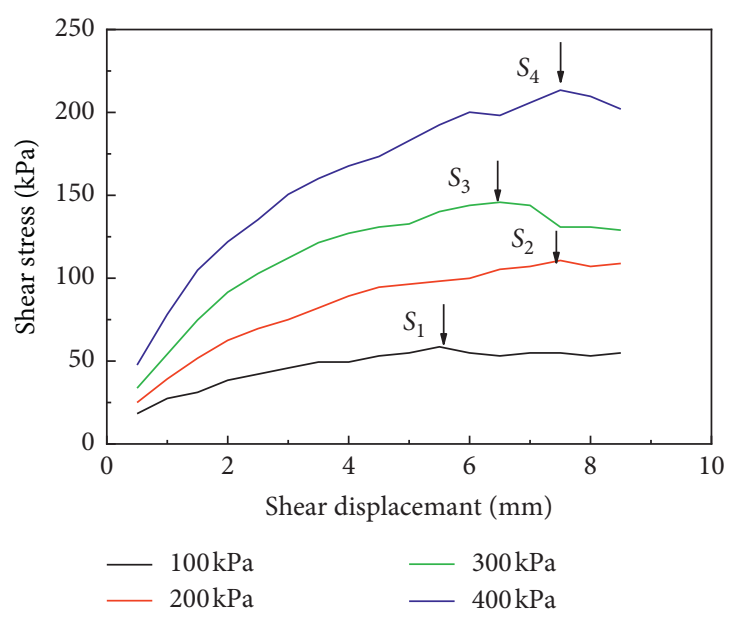

(a)

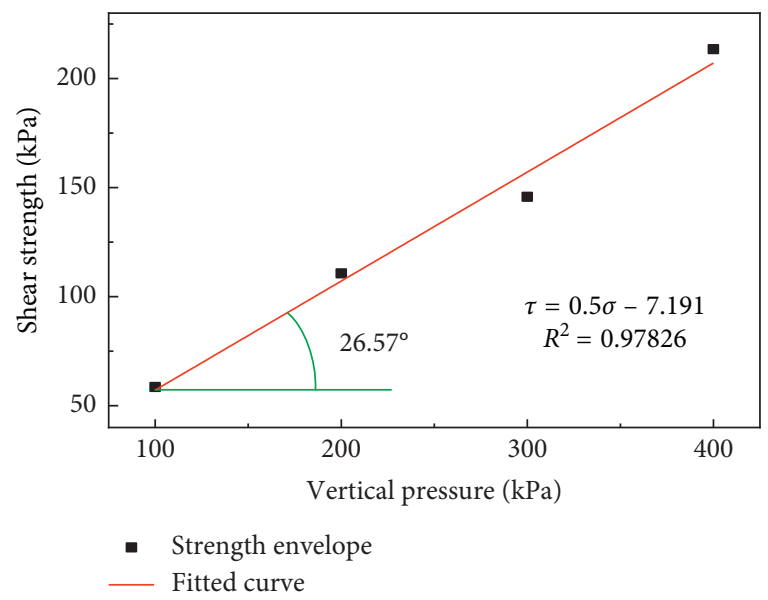

(b)

FIgUre 6: Analysis of nylon direct shear test results. (a) Shear force and shear displacement. (b) Vertical pressure and shear strength.

TABle 2: The macroscopic properties of nylon PA3200 powder.

\begin{tabular}{lc}
\hline Measurement parameters & Experiment results \\
\hline AOR $\left({ }^{\circ}\right)$ & 35.49 \\
Coefficient of internal friction & 0.50 \\
Angle of internal friction $\left({ }^{\circ}\right)$ & 26.57 \\
Particle size $(\mu \mathrm{m})$ & $20-120$
\end{tabular}

component of the particle; $\mathbf{V}_{i}$ is the translational velocity; $m$ is the mass of the particle; $\mathbf{M}_{i}$ is the out-of-balance moment due to the contacts; $i$ is the rotational velocity; $I$ is the rotational inertia of the particle; $\beta_{\mathrm{g}}$ is the global damping coefficient; and $d t$ is the time step.

3.1. van der Waals Force. Generally, the particles are devoid of polarity. However, due to the electronic movement of the molecules or atoms that make up the particles, particularly the molecules and atoms on the surface of the particles, the particles will have an instantaneous dipole. When the two particles are in close contact with each other, due to the 
action of the instantaneous dipole, the two particles will exert a mutual attraction force called van der Waals force between the particles. The nylon PA3200 powder used for sintering has a smaller particle size, and the force between the particles and the particles is far greater than the gravity, so the van der Waals force between the particles should be considered [23].

Hamaker [24] uses the principle of attraction potential and energy superposition of London-van der Waals to solve the gravitational potential between fine particles or between fine particles and walls by integration. It is shown as follows:

$$
U_{\mathrm{pp}}^{0}=\int_{V_{1}} \int_{V_{2}} \rho_{1} \rho_{2} U_{\mathrm{mm}} d V_{1} d V_{2}
$$

where the subscript pp represents particles; subscripts 1 and 2 represent particle 1 and particle 2 , respectively; $\rho_{1}$ and $\rho_{2}$ are the density of two particles $\left(\mathrm{kg} / \mathrm{m}^{3}\right)$; and $U_{\mathrm{mm}}$ is the total potential energy of the gravitational interaction between the molecules. By integrating the above equation, the gravitational potential energy between particles can be obtained:

$$
U_{\mathrm{pp}}^{0}=-\frac{\mathrm{Q}}{12 Z_{0}} \frac{d_{1} d_{2}}{d_{1}+d_{2}}
$$

where $d_{1}$ and $d_{2}$ are the diameters of the two particles $(m) ; Z_{0}$ is the distance between the particles $(m)$; and $Q$ is the Hamaker constant (eV).

The attraction between particle 1 and particle 2 , also the van der Waals force between particles, is

$$
F_{\mathrm{vdw}}^{0}=-\frac{\partial U_{\mathrm{pp}}^{0}}{\partial Z_{0}}=-\frac{Q}{12 Z_{0}^{2}} \frac{d_{1} d_{2}}{d_{1}+d_{2}},
$$

where the minus sign represents gravity and the negative sign is removed for convenience in the subsequent discussion.

When the particles are in contact with the plane, due to $d_{2} \rightarrow \infty$, the van der Waals force between the particles and the plane is

$$
F_{\mathrm{vdw}}^{0}=\frac{Q d}{12 Z_{0}^{2}}
$$

where $d$ is the diameter of the particle.

When the particle diameters of the two particles in contact with each other are equal, the van der Waals force between the particles is

$$
F_{\mathrm{vdw}}^{0}=\frac{Q d}{24 Z_{0}^{2}}
$$

3.2. Rolling Resistance Contact Model. Due to the certain agglomeration of nylon PA3200 powder, the rolling resistance contact model which is suitable for viscous materials and irregular granular materials was selected in this paper to describe the interaction between particles [25-27]. The rolling resistance contact model between particle-particle and particle-wall is shown in Figures 7 and 8, respectively.

The total rolling resistance torque relationship of the contact model is as follows:

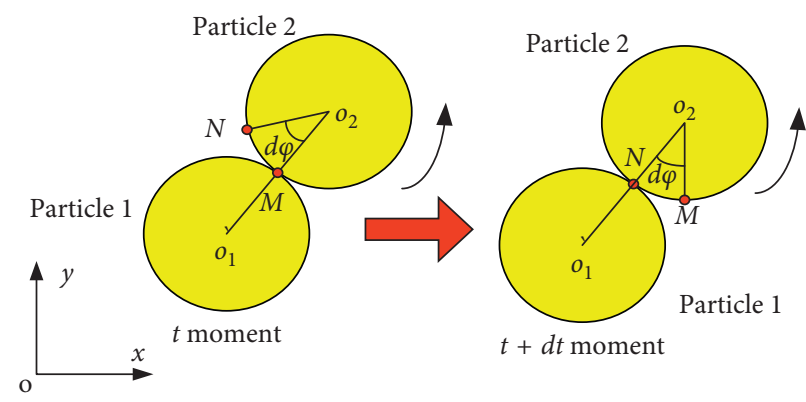

Figure 7: Particle-particle contact in the rolling resistance contact model.

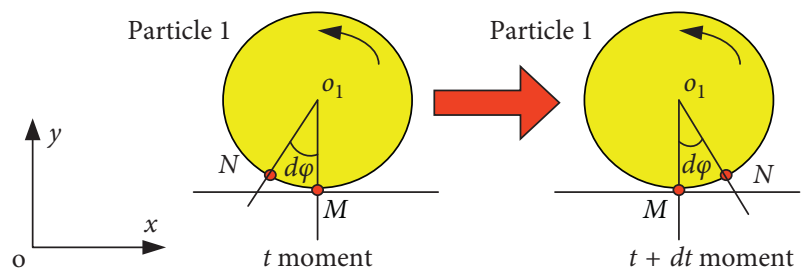

Figure 8: Particle-wall contact in the rolling resistance contact model.

$$
\mathbf{M}_{\mathrm{r}}=\mathbf{M}_{\mathrm{r}}^{\mathrm{k}}+\mathbf{M}_{\mathrm{r}}^{\mathrm{d}}
$$

where $\mathbf{M}_{\mathrm{r}}^{\mathrm{k}}$ is the spring torque and $\mathbf{M}_{\mathrm{r}}^{\mathrm{d}}$ is the viscous damping torque. The spring torque can be calculated as follows:

$$
\begin{aligned}
\Delta \mathbf{M}_{\mathrm{r}}^{\mathrm{k}} & =-k_{\mathrm{r}} \Delta \boldsymbol{\theta}_{\mathrm{r}}, \\
k_{\mathrm{r}} & =k_{\mathrm{s}} \bar{R}^{2},
\end{aligned}
$$

where $k_{\mathrm{r}}$ is the rolling resistance stiffness, $\bar{R}$ is the contact effective radius, and $\Delta \boldsymbol{\theta}_{\mathrm{r}}$ is the relative bend-rotation increment.

$$
\frac{1}{\bar{R}}=\frac{1}{R^{(1)}}+\frac{1}{R^{(2)}} .
$$

The magnitude of the updated rolling resistance moment is then checked against a threshold limit:

$$
\mathbf{M}_{\mathrm{r}}= \begin{cases}\mathbf{M}_{\mathrm{r}}, & \left\|\mathbf{M}_{\mathrm{r}}\right\| \leq \mathbf{M}_{\mathrm{r}}^{\mathrm{m}}, \\ \mathbf{M}_{\mathrm{r}}^{\mathrm{m}}\left(\frac{\mathbf{M}_{\mathrm{r}}}{\left\|\mathbf{M}_{\mathrm{r}}\right\|}\right), & \text { otherwise. }\end{cases}
$$

The limiting torque is defined as

$$
\mathbf{M}_{\mathrm{r}}^{\mathrm{m}}=\mu_{\mathrm{r}} \bar{R} \mathbf{F}_{\mathrm{n}},
$$

where $\mathbf{M}_{\mathrm{r}}^{k}$ is the conditional torque, $\mu_{\mathrm{r}}$ is the rolling friction coefficient, and $\mathbf{F}_{\mathrm{n}}$ is the normal force.

The relationship between the viscous rolling resistance coefficients can be expressed as

$$
C_{\mathrm{r}}=\eta_{\mathrm{r}} C_{\mathrm{r}}^{\mathrm{crit}}
$$

where $\eta_{\mathrm{r}}$ is the rolling viscous damping ratio and $C_{\mathrm{r}}^{\text {crit }}$ is the rolling critical viscous damping constant. 


$$
C_{\mathrm{r}}^{\mathrm{crit}}=\sqrt{I_{\mathrm{r}} K_{\mathrm{r}}}
$$

where $I_{\mathrm{r}}$ is the equivalent rotational inertia of the contact point between two particles.

$$
I_{\mathrm{r}}=\frac{1}{\left(\left(1 /\left(I_{1}+m_{1} r_{1}^{2}\right)\right)+\left(1 /\left(I_{2}+m_{2} r_{2}^{2}\right)\right)\right)},
$$

where $I_{1}$ and $I_{2}$ are the rotational inertia of particles 1 and 2, respectively; $m_{1}$ and $m_{2}$ are the mass for particles 1 and 2, respectively.

Based on the selection of the rolling resistance contact model in the PFC software, the Fish contact function was used to add a van der Waals force into the particle's contact.

\section{DEM Simulation}

4.1. Parameter Calibration of Nylon PA3200 Powder Contact Model. In this paper, the PFC software is used to model the entire AOR angle by adjusting various parameters [28] to obtain the characteristics of the AOR. Firstly, the microscopic parameters of rolling resistance contact model are initially assigned, and then a large number of debugging operations are performed on the different values of each parameter. The macroscopic physical and mechanical properties obtained by the AOR experiment are compared with the experimental results. In this process, several model parameters are involved, such as friction coefficient, stiffness ratio, porosity, and effective modulus. The specific physical and mechanical properties can be obtained when any set of parameters is selected from a given model, and the microscopic parameters of the DEM used are reasonably selected according to the degree of coincidence. It can be seen from Figure 2 that the powder has an irregular Gaussian distribution. However, the Gaussian distribution command in PFC simulation is regular. In order to be close to the actual situation, the particle size of the simulation experiment in this paper is set as 20 to $100 \mu \mathrm{m}$.

The common AOR model includes the cylinder model, the funnel model, and L-box model [29]. In this paper, the cylinder model is used to study the AOR of nylon PA3200 powder. To reduce the scale of calculation, this paper selects an axial section plane of the cylinder body for two-dimensional simulation, as shown in Figure 9. In Figure 9, $D$ is the diameter of the cylinder, $H$ is the height of the cylinder, $L$ is the diameter of the bottom plate, and $V$ is the lifting speed of the cylinder.

In previous studies, there is usually a requirement for the cross-sectional dimension, that is, the diameter of the cylinder should be selected to be more than 4 times the maximum particle size of the particle, and the height is 3 times the diameter of the cylinder. Therefore, in the subsequent numerical simulation, the diameter of the cylinder $D$, the height of the cylinder $H$, and the diameter of the bottom plate $L$ were selected to be $2 \mathrm{~mm}, 6 \mathrm{~mm}$, and $4 \mathrm{~mm}$, respectively, as the cylindrical model size value. When simulating the fluidity of the powder, a certain amount of particles was generated in the cylinder, and then the cylinder was lifted at a speed of $6 \mathrm{~mm} / \mathrm{s}$, and the particles were piled up to form a certain angle. Finally, the program of Fish was

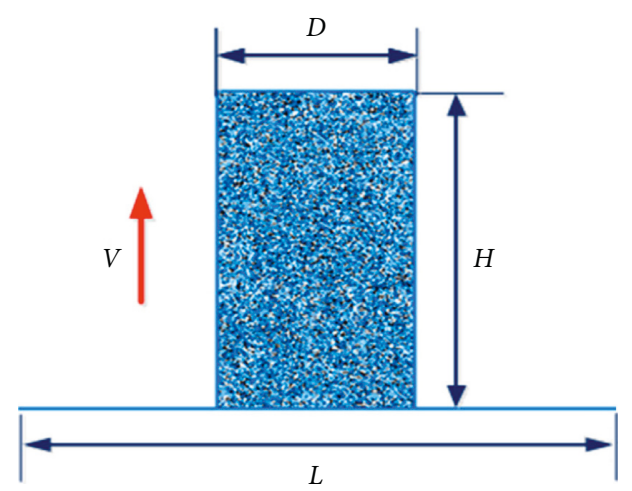

Figure 9: Cylinder model used in the DEM simulation.

written in the PFC software to calculate the position coordinates of the outermost particles and draw the contour curve of the outer particles. The contour curve formed by the particles is used to find the AOR. Figure 10 is a plot of the AOR.

Based on the AOR model, the rolling resistance coefficient between nylon PA3200 powder is continuously changed based on the initial value. The simulation results are shown in Table 3, and the outer surface contour curves of particle accumulation under different the rolling resistance coefficients are obtained as shown in Figure 11(a). It should be noted that the parameter change is adjusted based on the preliminary assignment. If the simulation result of the initial assignment differs greatly from the experimental result, the selection of the parameter range is expanded. On the contrary, the nearest selection approximates the experimental value. The AOR of the different curves in Figure 11(a) is extracted, and the AOR shown in Figure 11(b) is obtained. It can be seen that as the rolling resistance coefficient increases, the AOR increases. It can be seen from Table 3 that when the rolling resistance coefficient is 0.5 , the AOR of the numerical simulation is $35.3^{\circ}$, which is close to the experimental value of $35.49^{\circ}$. This is because the increase in the rolling resistance coefficient makes the free flow between particles to overcome greater resistance. Therefore, the rolling resistance coefficient in the simulation is set to 0.5 .

For particle model tests, the friction coefficient between particles is a vital factor. On the basis of the initial value, the friction coefficient between nylon PA3200 powders was changed constantly. The simulation results are shown in Table 4 . The influence of the friction coefficient between the particles on the macroscopic AOR is shown in Figure 12.

It can be seen from Figure 12 that the AOR increases from the increase in the friction coefficient. This is because the friction coefficient increases resulting in an increase in the frictional resistance of the particles, and slippage between the particles is more difficult. According to Table 4, when the friction coefficient is set to 0.5 , the numerical simulation results that the AOR $35.28^{\circ}$ is closer to the experimental value of $35.32^{\circ}$, so the value of 0.5 is selected as the friction coefficient in the simulation.

4.2. Parameter Calibration Results. With the understanding of the influence of model microscopic parameters on 


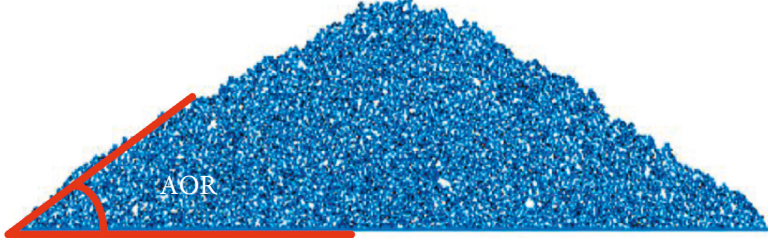

FIgURE 10: AOR accumulation.

TABLE 3: Calibration of the rolling resistance coefficient.

\begin{tabular}{lc}
\hline Rolling resistance coefficient & AOR $\left(^{\circ}\right)$ \\
\hline 0.40 & 32.60 \\
0.50 & 35.30 \\
0.60 & 35.87 \\
0.70 & 36.11 \\
0.80 & 37.12 \\
\hline
\end{tabular}

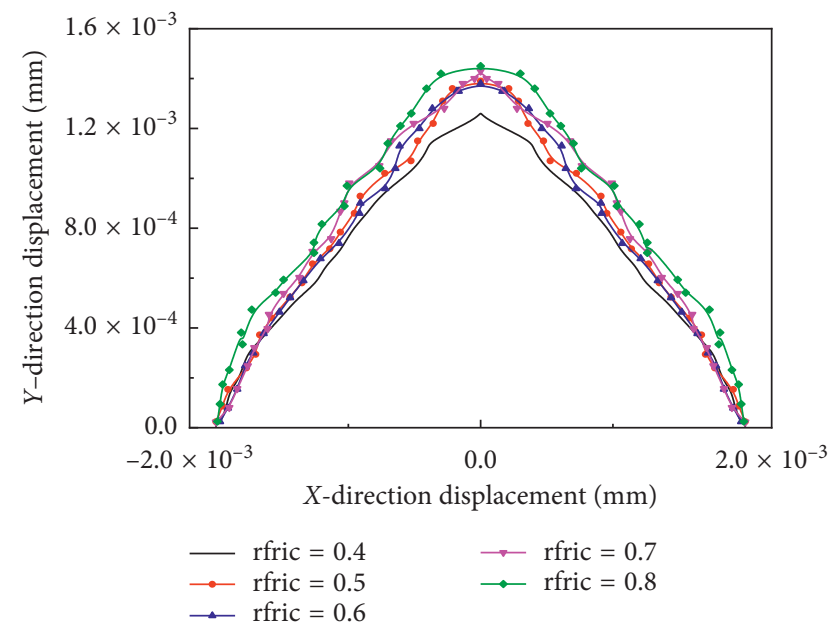

(a)

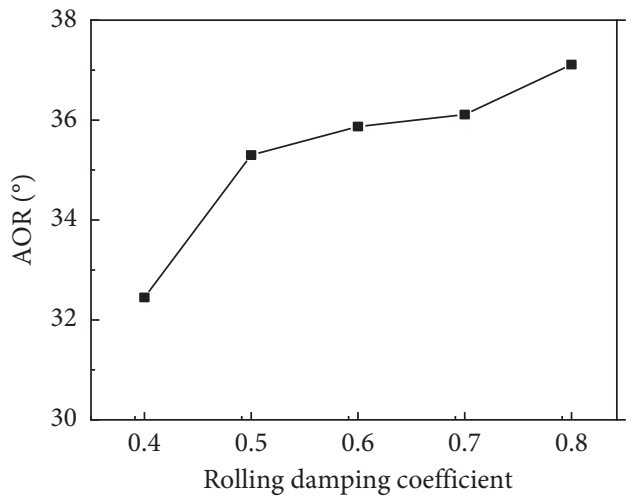

(b)

FIGURE 11: Effect of the rolling resistance coefficient on the AOR. (a) Particle projection outer surface contour curve. (b) Relationship between the rolling resistance coefficient and AOR.

macroscopic properties, combined with the actual results of the AOR experiment, the microscopic parameters of the model were calibrated, and the results of the experiment and simulation are shown in Table 5.
TABLE 4: Relationship between the friction coefficient and the AOR.

\begin{tabular}{lc}
\hline Friction coefficient & AOR $\left(^{\circ}\right)$ \\
\hline 0.40 & 34.93 \\
0.45 & 35.00 \\
0.50 & 35.28 \\
0.55 & 36.87 \\
0.60 & 37.69 \\
\hline
\end{tabular}

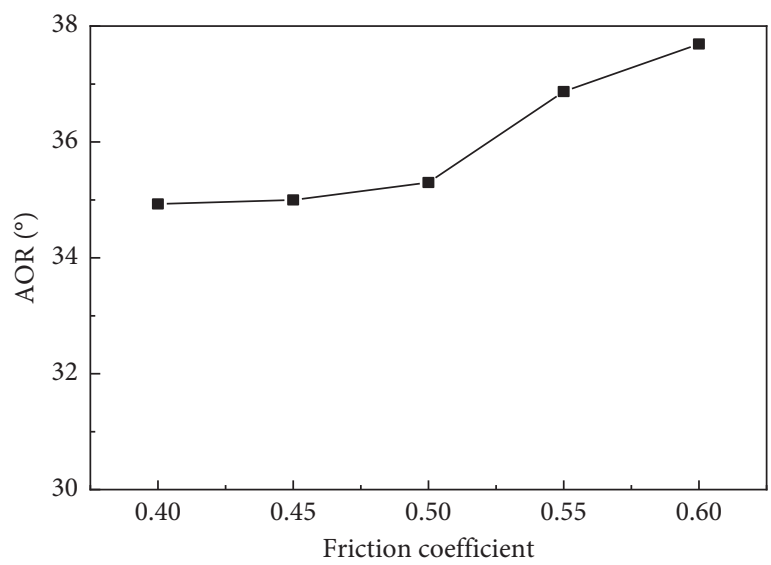

FIGURE 12: Effect of friction coefficient between particles on the AOR.

TABLE 5: Microscopic parameters of nylon PA3200 powder fluidity model.

\begin{tabular}{lc}
\hline Contact parameter & Value \\
\hline Effective modulus $(\mathrm{Pa})$ & $1 e 7$ \\
Stiffness ratio & 1.50 \\
Nylon and nylon friction coefficient & 0.50 \\
Nylon and wall friction coefficient & 0.50 \\
Rolling resistance coefficient & 0.50 \\
Porosity & 0.30 \\
Particle density $\left(\mathrm{g} / \mathrm{cm}^{3}\right)$ & 1.00 \\
Particle size $(\mu \mathrm{m})$ & $20-100$ \\
\hline
\end{tabular}

\section{Analysis and Discussion}

5.1. Orthogonal Experiment Design. Orthogonal experiment design is a multifactor experimental design method based on the probability theory and mathematical statistics [30]. The orthogonal experiment can be used to obtain the optimal combination level and further to analyze the influencing factors. In this paper, the AOR model used in the previous calibration of microscopic parameters was used to study the factors affecting the fluidity of the powder. As shown in Table 6, the factors of orthogonal experiment design are particle size range (A) and the distribution type (B). The particle size is taken at four levels, which are $20-40 \mu \mathrm{m}$, $40-60 \mu \mathrm{m}, 60-80 \mu \mathrm{m}$, and $80-100 \mu \mathrm{m}$, respectively. The distribution type is taken at two levels, which are the uniform distribution and Gaussian distribution, respectively. A $\mathrm{L}_{8}\left(4 \times 2^{1}\right)$ orthogonal table was selected to carry out a twofactor mixed horizontal orthogonal experiment, as shown in Table 7. 
TABLE 6: Factors and levels of orthogonal experiment.

\begin{tabular}{lcc}
\hline \multirow{2}{*}{ Levels } & \multicolumn{2}{c}{ Factors } \\
& Particle size $(\mu \mathrm{m})(\mathrm{A})$ & Distribution type $(\mathrm{B})$ \\
\hline 1 & $20-40$ & Uniform distribution \\
2 & $40-60$ & Gaussian distribution \\
3 & $60-80$ & - \\
4 & $80-100$ & - \\
\hline
\end{tabular}

TABLE 7: Design of orthogonal experiment.

\begin{tabular}{lcc}
\hline Test number & \multicolumn{2}{c}{ Factors } \\
\hline 1 & A & B \\
2 & 1 & 1 \\
3 & 1 & 2 \\
4 & 2 & 1 \\
5 & 2 & 2 \\
6 & 3 & 1 \\
7 & 3 & 2 \\
8 & 4 & 1 \\
\hline
\end{tabular}

5.2. Range Analysis. The range analysis is one of the commonly used methods of orthogonal design, which is easy to understand and find the optimal level and optimal combination of primary and secondary factors of the test to meet the requirements of the general test quickly. A range analysis includes two critical parameters $K_{j i}$ and $R_{j} . K_{j i}$ is the average value of the test indicators corresponding to the level $i$ of column $j$, and $R_{j}$ is the difference between the maximum value and the minimum value of the average index value at each level of column $j$, which is the range of column $j$. The larger $R_{j}$, the greater the influence of this factor of the test indicators. The equations for calculating $K_{j i}$ and $R_{j}$ are shown below:

$$
\begin{aligned}
K_{j i} & =\frac{1}{N_{i}} \sum_{j=1}^{N_{i}} y_{j i}, \\
R_{j} & =\max \left(K_{j 1}, K_{j 2}, \ldots, K_{\mathrm{ji}}\right)-\min \left(K_{j 1}, K_{j 2}, \ldots, K_{\mathrm{ji}}\right),
\end{aligned}
$$

where $i$ is the level number $(i=1,2,3,4), j$ is the factor notation $(\mathrm{A}, \mathrm{B}), y_{j i}$ is one result value for factor $j$ at level $i$, and $N_{i}$ is the total number of levels.

According to the orthogonal experimental design scheme, the results from the above numerical simulation conditions are shown in Table 8 . It can be known that the difference between the two columns is different, indicating that the different levels of each factor have different effects on the AOR. The result is $R_{\mathrm{A}}>R_{\mathrm{B}}$, and it can be known that the particle size range $A$ has a greater influence on the AOR than the distribution type $\mathrm{B}$.

5.3. Analysis of Variance. Analysis of variance is a standard statistical technique used to determine the extent of influence, estimate the confidence level of the influence, and evaluate the experimental errors. In the analysis of variance, an $F$ value of each factor represents the ratio of the sum of the
TABLE 8: Results of orthogonal experiment design.

\begin{tabular}{lccc}
\hline Test number & $\begin{array}{c}\text { Particle size }(\mu \mathrm{m}) \\
(\mathrm{A})\end{array}$ & $\begin{array}{c}\text { Distribution type } \\
(\mathrm{B})\end{array}$ & AOR $\left(^{\circ}\right)$ \\
\hline 1 & 1 & 1 & 32.82 \\
2 & 1 & 2 & 33.35 \\
3 & 2 & 1 & 32.29 \\
4 & 2 & 2 & 33.10 \\
5 & 3 & 1 & 32.82 \\
6 & 3 & 2 & 31.76 \\
7 & 4 & 1 & 34.25 \\
8 & 4 & 2 & 35.56 \\
\hline$K_{j 1}$ & 33.08 & 33.05 & \\
$K_{j 2}$ & 32.70 & 33.44 & \\
$K_{j 3}$ & 32.29 & & \\
$K_{j 4}$ & 34.91 & & \\
$R_{j}$ & 2.62 & 0.39 & \\
\hline
\end{tabular}

square of each factor's average deviation from that of the experimental error. The calculation of the $F$ value demands the sum of square deviation from each factor $\left(\mathrm{SS}_{j}\right)$, the total sum of the squared deviation $\left(\mathrm{SS}_{T}\right)$, the degree of freedom of the factor $j(f)$, and the total degree of freedom of the experiments $\left(f_{T}\right) \cdot f_{j}$ is equal to the number of levels of factor $j$ minus 1 while $f_{T}$ is the total number of experiments minus 1 . Thus, the degree of freedom of the experimental error $f_{e}$ is the difference between $f_{T}$ and the sum of the $f$ of all the factors.

$\mathrm{SS}_{j}$ of the factor $j$ and $\mathrm{SS}_{T}$ are calculated using the following equations:

$$
\begin{aligned}
& \mathrm{SS}_{j}=\frac{1}{n} \sum_{i=1}^{n} k_{j i}^{2}-\frac{\left(\sum_{i=1}^{n^{2}} Y_{i}\right)^{2}}{n^{2}} \\
& \mathrm{SS}_{T}=\sum_{i=1}^{m} Y_{i}^{2}-\frac{1}{m}\left(\sum_{i=1}^{m} Y_{i}\right)^{2} \\
& \mathrm{SS}_{e}=\mathrm{SS}_{T}-\mathrm{SS}_{j}
\end{aligned}
$$

where $n$ is the number of levels of factor $j ; m$ is the number of factors; and $Y_{i}$ is the value of the result of the level $i$.

The results of the analysis of variance are shown in Table 9. The $F$ value is compared to the value of $F_{\alpha}\left(f, f_{e}\right)$, which indicates the threshold $F$ values with the risk level $\alpha$ (confidence level $=1-\alpha$ ), where $f$ and $f_{e}$ are the degrees of freedom associated to the numerator and denominator, respectively. In this study, the factor $\mathrm{A}$ is $F_{\alpha}(3,4)$ and factor $\mathrm{B}$ is $F_{\alpha}(1,6)$. By referring to the distribution table of the $F$ value, the significance level is selected, and the corresponding critical value is found for comparison to determine whether the factor has a significant degree of influence. For factor A, $F_{0.05}(3,4)=6.59, F_{0.1}(3,4)=4.19$, and $F_{0.1}(3,4)$ $<F_{\mathrm{A}}<F_{0.05}(3,4)$, and then factor $\mathrm{A}$ is significant at the level of $\alpha=0.1$ and has a significant influence. For factor $\mathrm{B}, F_{0.25}$ $(1,6)=1.62$, and $F_{\mathrm{B}}<F_{0.25}(1,6)$ indicates that the influence of factors is not significant. Therefore, the results of the analysis of variance are consistent with the range analysis, and the particle size has a greater influence on the AOR than the distribution type. 
TABLE 9: Analysis of variance.

\begin{tabular}{lcccc}
\hline Source & SS & $f$ & MS & $F$ \\
\hline A & 8.0174 & 3 & 2.6725 & 5.08 \\
B & 0.312 & 1 & 0.312 & 0.59 \\
$e$ & 1.5785 & 3 & 0.5262 & \\
$T$ & 9.9079 & 7 & & \\
\hline
\end{tabular}

${ }^{*} S S$ : adjusted sums of squares; $f$ : degree of freedom; MS: adjusted mean squares; F: sum of square deviation; $e$ : error; $T$ : total.

\section{Conclusions}

In this paper, an experimental and numerical simulation study was carried out on the powder fluidity affecting the powder paving quality during the selective laser sintering of nylon PA3200 powder. The related studies were carried out from the PSD range and the distribution type, and the main conclusions are shown below:

(1) The AOR, angle of internal friction, and the friction coefficient of nylon PA3200 were obtained by the experimental method. The rolling resistance coefficient and friction coefficient within the contact model were calibrated by the results of the AOR experiments.

(2) Based on simulation of a series of different parameters (such as particle size and PSD) in combination with the orthogonal experimental method, it can be seen that the PSD has a stronger influence on the AOR than particle size, and the fluidity of uniform distribution is better than that of the Gaussian distribution.

\section{Data Availability}

The data used to support the findings of this study are available from the corresponding author upon request.

\section{Conflicts of Interest}

The authors declare that they have no conflicts of interest.

\section{Acknowledgments}

This study was supported by the Scientific Research Fund of Hunan Provincial Education Department (18B074 and 18C0092), the National Natural Science Foundation of China (51605409, 51605410, 11772135, and 51775468), the National Independent Innovation Demonstration Area Foundation of Changsha Zhuzhou Xiangtan (2018XK2302 and 2017XK2107), and the Hunan Provincial Natural Science Foundation of China (2019JJ20015).

\section{References}

[1] H. Guo, R. Lv, and S. Bai, "Recent advances on 3D printing graphene-based composites," Nano Materials Science, vol. 1, no. 2, pp. 101-115, 2019.

[2] Q. Zhou, B. Armstrong, I. Larson, P. J. Stewart, and D. A. V. Morton, "Improving powder flow properties of a cohesive lactose monohydrate powder by intensive mechanical dry coating," Journal of Pharmaceutical Sciences, vol. 99, no. 2, pp. 969-981, 2010.
[3] S. Yang and J. R. G. Evans, "Metering and dispensing of powder; the quest for new solid freeforming techniques," Powder Technology, vol. 178, no. 1, pp. 56-72, 2007.

[4] H. P. Goh, P. W. S. Heng, and C. V. Liew, "Comparative evaluation of powder flow parameters with reference to particle size and shape," International Journal of Pharmaceutics, vol. 547, no. 1-2, pp. 133-141, 2018.

[5] H. Zhang, B. Xiong, X. An, C. Ke, and G. Wei, "Prediction on drag force and heat transfer of spheroids in supercritical water: a PR-DNS study," Powder Technology, vol. 342, pp. 99-107, 2019.

[6] H. Zhang, B. Xiong, X. An, C. Ke, and G. Wei, "Numerical investigation on the effect of the incident angle on momentum and heat transfer of spheroids in supercritical water," Computers \& Fluids, vol. 179, pp. 533-542, 2019.

[7] G. Wei, H. Zhang, X. An, B. Xiong, and S. Jiang, "CFD-DEM study on heat transfer characteristics and microstructure of the blast furnace raceway with ellipsoidal particles," Powder Technology, vol. 346, pp. 350-362, 2019.

[8] L. Dai, V. Sorkin, G. Vastola, and Y. W. Zhang, "Dynamics calibration of particle sandpile packing characteristics via discrete element method," Powder Technology, vol. 347, pp. 220-226, 2019.

[9] A. Maćias-Garćia, E. M. Cuerda-Correa, and M. A. Díra-D'iez, "Application of the Rosin-Rammler and Gates-GaudinSchuhmann models to the particle size distribution analysis of agglomerated cork," Materials Characterization, vol. 52, no. 2, pp. 159-164, 2004.

[10] J. J. Fitzpatrick, S. A. Barringer, and T. Iqbal, "Flow property measurement of food powders and sensitivity of Jenike's hopper design methodology to the measured values," Journal of Food Engineering, vol. 61, no. 3, pp. 399-405, 2004.

[11] A. B. Yu, C. L. Feng, R. P. Zou, and R. Y. Yang, "On the relationship between porosity and interparticle forces," Powder Technology, vol. 130, no. 1-3, pp. 70-76, 2003.

[12] J. L. Amorós, G. Mallol, C. Feliu, and M. J. Orts, "Study of the rheological behaviour of monomodal quartz particle beds under stress. A model for the shear yield functions of powders," Chemical Engineering Science, vol. 66, no. 18, pp. 4070-4077, 2011.

[13] W. Wang, J. Zhang, S. Yang, H. Zhang, H. Yang, and G. Yue, "Experimental study on the angle of repose of pulverized coal," Particuology, vol. 8, no. 5, pp. 482-485, 2010.

[14] H. M. B. Al-Hashemi and O. S. B. Al-Amoudi, "A review on the angle of repose of granular materials," Powder Technology, vol. 330, pp. 397-417, 2018.

[15] D. Geldart, E. C. Abdullah, A. Hassanpour, L. C. Nwoke, and I. Wouters, "Characterization of powder flowability using measurement of angle of repose," China Particuology, vol. 4, no. 3-4, pp. 104-107, 2006.

[16] E. C. Abdullah and D. Geldart, "The use of bulk density measurements as flowability indicators," Powder Technology, vol. 102, no. 2, pp. 151-165, 1999.

[17] R. L. Carr, "Evaluating flow properties of solids," Chemical Engineering, vol. 72, no. 2, pp. 163-168, 1965.

[18] E. Guerin, P. Tchoreloff, B. Leclerc, D. Tanguy, M. Deleuil, and G. Couarraze, "Rheological characterization of pharmaceutical powders using tap testing, shear cell and mercury porosimeter," International Journal of Pharmaceutics, vol. 189, no. 1, pp. 91-103, 1999.

[19] A. W. Jenike, "A measure of flowability for powders and other bulk solids," Powder Technology, vol. 11, no. 1, pp. 89-90, 1975. 
[20] P. A. Cundall and O. D. L. Strack, "A discrete numerical model for granular assemblies," Geotechnique, vol. 29, no. 1, pp. 47-65, 1979.

[21] R. Balevičius, R. Kačianauskas, Z. Mróz, and I. Sielamowicz, "Analysis and DEM simulation of granular material flow patterns in hopper models of different shapes," Advanced Powder Technology, vol. 22, no. 2, pp. 226-235, 2011.

[22] S. Jiang, Y. Ye, Y. Tan et al., "Discrete element simulation of particle motion in ball mills based on similarity," Powder Technology, vol. 335, pp. 91-102, 2018.

[23] H. P. Zhu, Z. Y. Zhou, R. Y. Yang, and A. B. Yu, "Discrete particle simulation of particulate systems: theoretical developments," Chemical Engineering Science, vol. 62, no. 13, pp. 3378-3396, 2007.

[24] H. C. Hamaker, "The London-Van der Waals attraction between spherical particles," Physica, vol. 4, no. 10, pp. 1058-1072, 1937.

[25] J. Ai, J.-F. Chen, J. M. Rotter, and J. Y. Ooi, "Assessment of rolling resistance models in discrete element simulations," Powder Technology, vol. 206, no. 3, pp. 269-282, 2011.

[26] K. Iwashita and M. Oda, "Rolling resistance at contacts in simulation of shear band development by DEM," Journal of Engineering Mechanics, vol. 124, no. 3, pp. 285-292, 1998.

[27] M. J. Jiang, H.-S. Yu, and D. Harris, "A novel discrete model for granular material incorporating rolling resistance," Computers and Geotechnics, vol. 32, no. 5, pp. 340-357, 2005.

[28] C. J. Coetzee, "Review: calibration of the discrete element method," Powder Technology, vol. 310, pp. 104-142, 2017.

[29] S. Q. Jiang, Y. Ye, M. X. He et al., "Mixing uniformity of irregular sand and gravel materials in a rotating drum with determination of contact model parameters," Powder Technology, vol. 354, pp. 377-391, 2019.

[30] R. Liu, Y. Zhang, C. Wen, and J. Tang, "Study on the design and analysis methods of orthogonal experiment," Experimental Technology and Management, vol. 27, no. 11, pp. 52$55,2010$. 


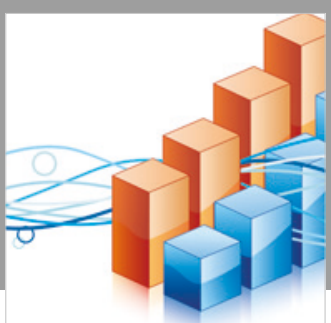

Advances in

Operations Research

\section{-n-m}
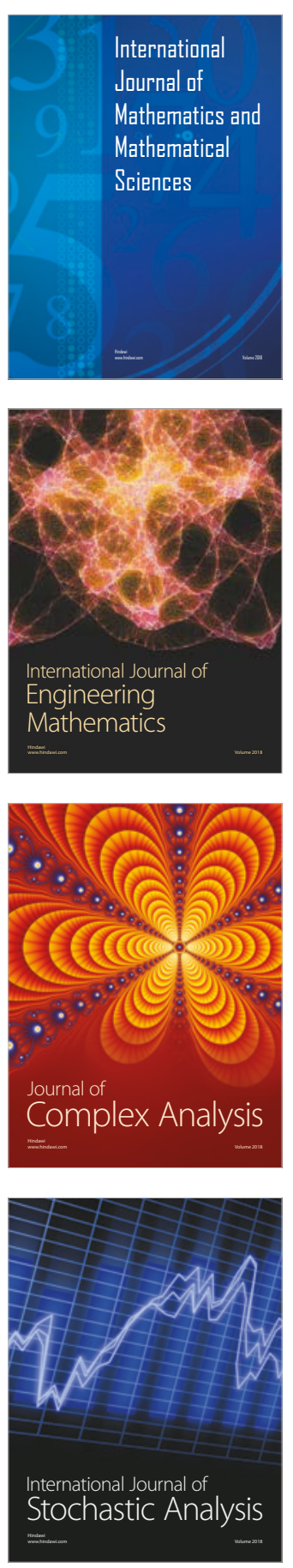
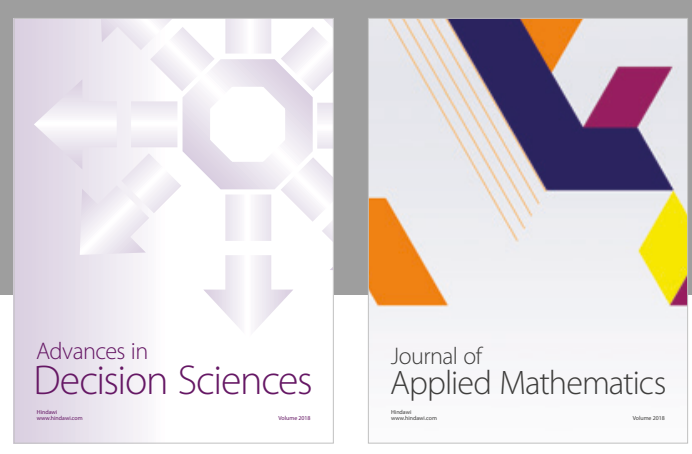

Journal of

Applied Mathematics
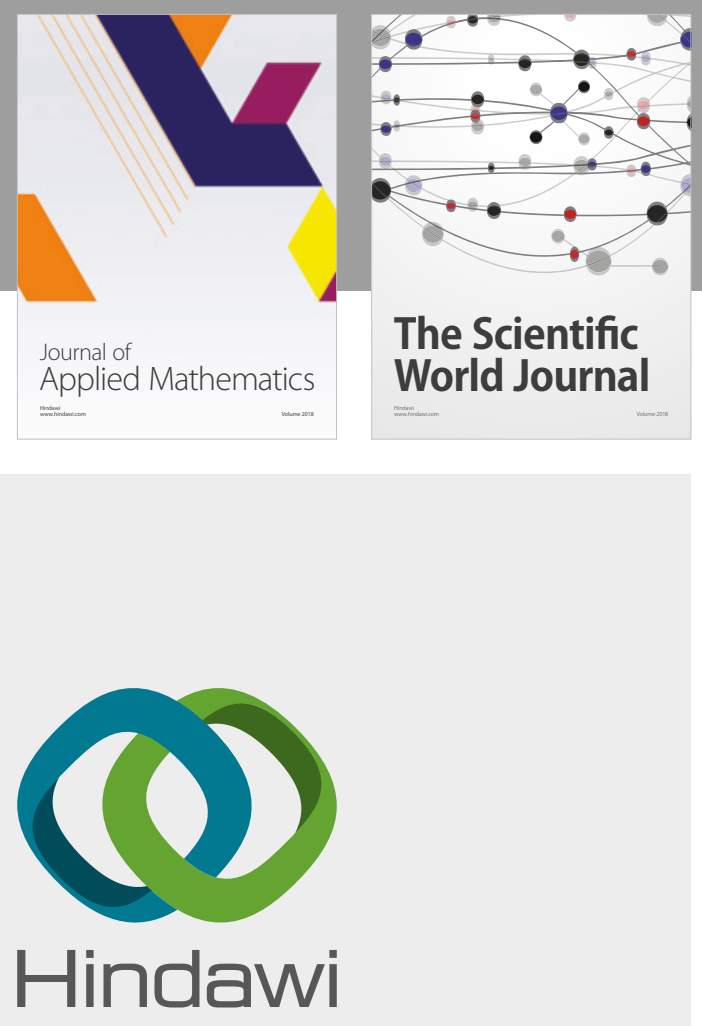

Submit your manuscripts at

www.hindawi.com

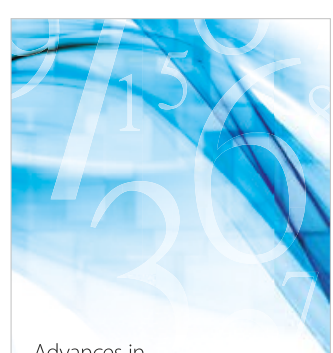

Advances in
Numerical Analysis
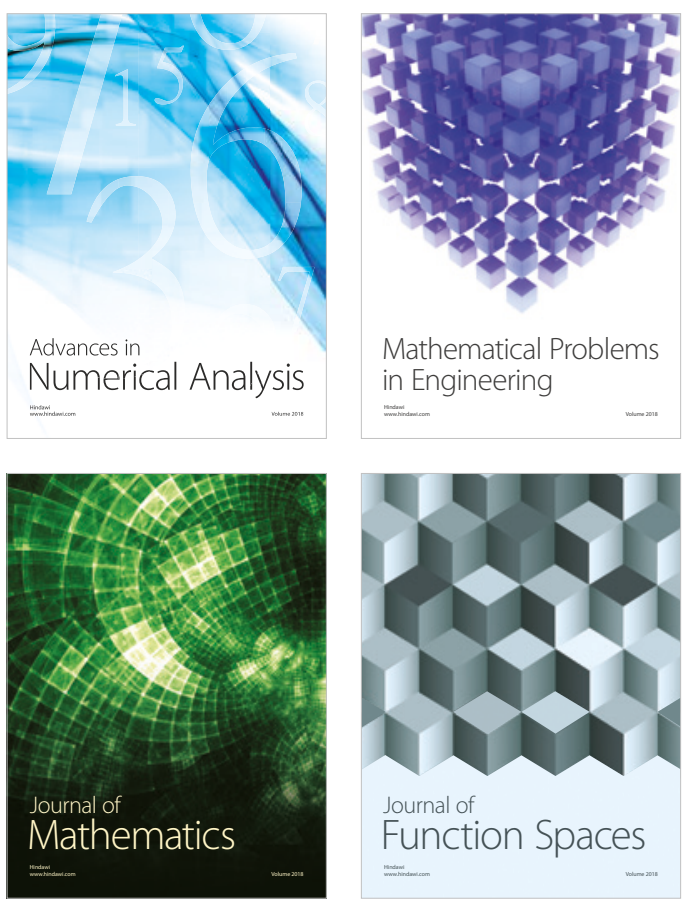

Mathematical Problems in Engineering

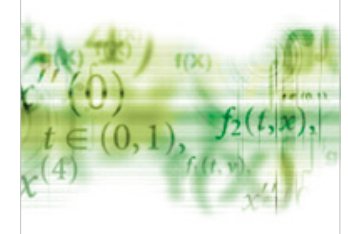

International Journal of

Differential Equations

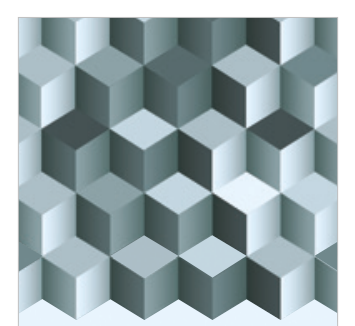

Journal of

Function Spaces

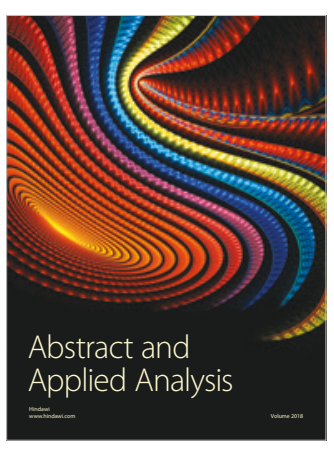

The Scientific

World Journal

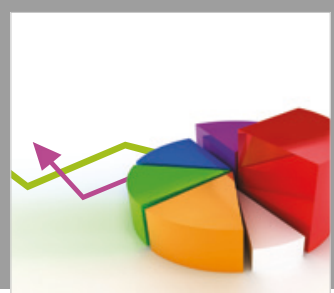

Journal of

Probability and Statistics
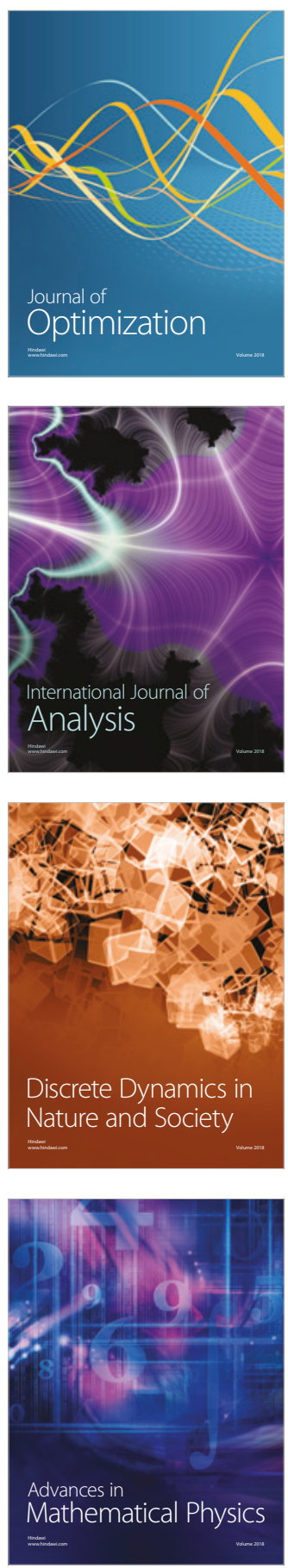